\title{
Enhanced endothelial cell density on NiTi surfaces with sub-micron to nanometer roughness
}

\author{
Harry D Samaroo \\ Jing Lu \\ Thomas J Webster \\ Division of Engineering, Brown \\ University, Providence, RI, USA
}

Correspondence:Thomas J Webster

Division of Engineering, Brown University,

Providence, RI, 02917, USA

$\mathrm{Tel}+\mathrm{I} 4018632318$

Fax +1 401 8639107

Email thomas_webster@brown.edu

\begin{abstract}
The shape memory effect and superelastic properties of NiTi (or Nitinol, a nickel-titanium alloy) have already attracted much attention for various biomedical applications (such as vascular stents, orthodontic wires, orthopedic implants, etc). However, for vascular stents, conventional approaches have required coating NiTi with anti-thrombogenic or antiinflammatory drug-eluting polymers which as of late have proven problematic for healing atherosclerotic blood vessels. Instead of focusing on the use of drug-eluting anti-thrombogenic or anti-inflammatory proteins, this study focused on promoting the formation of a natural antithrombogenic and anti-inflammatory surface on metallic stents: the endothelium. In this study, we synthesized various NiTi substrates with different micron to nanometer surface roughness by using dissimilar dimensions of constituent NiTi powder. Endothelial cell adhesion on these compacts was compared with conventional commercially pure (cp) titanium (Ti) samples. The results after 5 hrs showed that endothelial cells adhered much better on fine grain $(<60 \mu \mathrm{m})$ compared with coarse grain NiTi compacts $(<100 \mu \mathrm{m})$. Coarse grain NiTi compacts and conventional Ti promoted similar levels of endothelial cell adhesion. In addition, cells proliferated more after 5 days on NiTi with greater sub-micron and nanoscale surface roughness compared with coarse grain NiTi. In this manner, this study emphasized the positive pole that NiTi with sub-micron to nanometer surface features can play in promoting a natural anti-thrombogenic and anti-inflammatory surface (the endothelium) on a vascular stent and, thus, suggests that more studies should be conducted on NiTi with sub-micron to nanometer surface features.
\end{abstract}

Keywords: nitinol, stents, nanophase, surface roughness, adhesion, endothelial cells

\section{Introduction}

When blood vessels become occluded due to atherosclerosis, balloon angioplasty has been used as an easy, common treatment to clear the vessel allowing for blood flow. This procedure involves the use of an expanding balloon which ruptures the plaque at the occluded site to restore normal blood flow. However, products from the ruptured plaque may induce blood clotting postoperatively, and the artery may eventually reocclude (McDougall 2006).

To ameliorate this problem, percutaneous coronary intervention (PCI) has been used and arteries have been propped open after angioplasty by the implantation of a metallic vascular stent (McDougall 2006). However, neointimal hyperplasia is a serious problem after stent deployment and is due to a complex combination of several factors including excessive smooth muscle cell growth induced by stress and strain imbalances at the stent-vessel interface (Armitage et al 2003). Moreover, oscillating shear stresses caused by blood flow disruption at the stent-vessel interface have been implicated in altering vascular cell responses leading to neointimal hyperplasia (Armitage et al 2003). Inflammation (mediated by activated monocytes and macrophages) and thrombosis (mediated by activated platelets) have also led to problems with vascular stents. 
For this reason, some time ago, researchers turned their attention towards coating metallic stents with antithrombogenic and anti-inflammatory drug-eluting polymers. However, conventional approaches of coating metal stents with drug-eluting polymers have lately proven problematic in healing atherosclerotic blood vessels. Consequently, through the use of drug-eluting stents, patients have faced an increased risk of blood clots which may block arteries and further lead to heart attacks and death.

The failure of drug-eluting stents has led researchers to turn their attention towards novel materials and surface treatments to enhance endothelial cell adhesion and function while preventing restenosis. Due to advances in nanotechnology, some scientists have examined nanotextured stent surfaces to promote rapid surface coverage with an endothelial monolayer. Such a phenomenon will not only protect the stent surface from inflammatory cells but it will also have anti-thrombogenic effects and prevent the proliferation of smooth muscle cells that cause restenosis. As an example of this research, Choudhary and colleagues (2006) first demonstrated the benefits of nanostructured titanium (Ti) and CoCrMo vascular stents on increasing the selectively of endothelial cell adhesion. Recently, they also showed enhanced endothelial cell functions (including collagen synthesis) on nanostructured compared with nano-smooth Ti. Moreover, Miller and colleagues (2004) reported increased endothelial cell functions on polymers (such as poly-lacticco-glycolic acid [PLGA], polyetherurethane [PU], and polycaprolactone $[\mathrm{PCL}]$ ) with nanostructured compared to nano-smooth surface features. These studies hypothesized that a sub-micron/nanometer topography (whether on polymers or metals) mimics the structure of natural vascular tissue (which includes nanostructured extracellular matrix proteins such as collagen and elastin) better than currently used, nano-smooth vascular stents. However, to date, there have been little to no studies on modifying the degree of sub-micron to nanometer roughness for the key vascular stent material NiTi (Nitinol or a nickel-titanium alloy).

In this study, we investigate the benefits of sub-micron and nanometer surface features on NiTi for vascular stent applications. The shape memory effect and superelastic properties of NiTi have already attracted much attention for various biomedical applications (such as orthodontic wires, orthopedic implants, eyeglass frames, etc). NiTi has both mechanical and thermal shape memory properties, and thus, a NiTi vascular stent can unfold once exposed to the convective heat flow of blood. The superelastic property of NiTi provides a high level of flexibility that is advantageous for delivering the stent to the occluded vascular site (Shabalovskaya et al 2002; Yeh et al 2006). However, the ability of conventional NiTi to rapidly endothelialize upon implantation has been less than satisfactory; hence, the need for rapid endothelialization has prompted the current investigation into the benefits of NiTi with sub-micron to nanometer surface features.

To develop the next generation of NiTi stents, in this study, we synthesized NiTi substrates with different micron to nanometer surface roughness by using various constituent dimensions of NiTi powder. Previous studies have synthesized nanostructured NiTi by shock compression of mechanically amorphized NiTi powder (Xu et al 2004). However, amorphized NiTi may lose its shape-memory properties advantageous for vascular stent applications. Other studies simply polished NiTi sheets with different grades of metallographic papers (Wirth et al 2004; Yeh et al 2006). However, polished NiTi surfaces have been shown to increase thrombogenicity, promoting clotting and neointima formation (Armitage 2003). In this study, we synthesized NiTi compacts of different roughness by compressing different dimensions of NiTi powder in a liquid nitrogen bath to induce deformation in the martensitic phase. Importantly, some have hypothesized that materials composed of nano compared with micron particles possess improved mechanical properties (such as flexibility, toughness, etc) (Siegel 1996); these improved mechanical properties of nano NiTi may be beneficial for reducing stress and strain imbalances as well as oscillating blood flows at the stent-vessel interface which leads to neointimal hyperplasia. We evaluated the cytocompatibility of these NiTi substrates by studying endothelial cell adhesion and cell proliferation in comparison with smooth conventional cp Ti substrates. In doing so, we elucidated a promising material (sub-micron to nanometer rough NiTi) for vascular stent applications.

\section{Materials and methods Substrate preparation}

NiTi powder was purchased from the Special Metals Company (New Hartford, NY) with a weight composition of $\mathrm{Ni}=55.5 \%$ and $\mathrm{Ti}=44.5 \%$ and with quoted particle sizes ranging from 10 microns to -80 mesh $(90 \%$ or more particles pass through a $177 \mu \mathrm{m}$ porous diameter sieve). Different dimensions of NiTi powder were obtained by sifting the stock powder through a 60 micron diameter opening stainless steel wire cloth. We derived a fine grain sample $(<60 \mu \mathrm{m})$ and a coarse grain stock sample $(<100 \mu \mathrm{m})$. Powders were loaded into a steel-tool die and all compact discs (diameter: $12 \mathrm{~mm}$, thickness: 1-2 mm) were produced using a uniaxial, single-ended compacting hydraulic press (Carver, Lafayette, IN). The pressure increased to 2-3 
GPa at intervals of 2 min to a maximum pressure of $8 \mathrm{GPa}$ over 7 minutes. Permanent deformation of NiTi in the martensitic (low-temperature) phase was achieved by compression in a liquid nitrogen bath (Figure 1A). Compacts were synthesized by systematically increasing the pressure followed by cooling in liquid nitrogen to emulate the NiTi stress-strain relation shown in Figure 1B. Before cell experiments, all NiTi compacts were placed in phosphate buffer saline (PBS) solution for 1 day and sterilized by ultra violet light exposure for 30 min on both sides. Ti substrates (Alfa Aesar) were sterilized by autoclaving and ultra violet light exposure for $30 \mathrm{~min}$ on both sides. Sterile tissue culture polystyrene coverslips (Fisher) were used as a reference substrate.

\section{Surface characterization}

The surfaces of the NiTi compacts were characterized for roughness using scanning electron microscopy (SEM; LEO 1530 VP). SEM images were used to estimate the average grain size of the different NiTi substrates. Surface chemistry of NiTi compacts were characterized using energy dispersive X-ray spectroscopy (EDS). Data on the surface composition of $\mathrm{Ni}(\mathrm{wt} \%)$ and $\mathrm{Ti}(\mathrm{wt} \%)$ were recorded and compared for all NiTi substrates to ensure that surface chemistry remained constant.

\section{Cell experiments}

Rat aortic endothelial cells ( $\mathrm{RAEC})(\mathrm{PN}=9)$ were purchased from VEC Technologies, cultured in MCDB-131 Complete Medium(VEC Technologies; Rensselaer, NY) under standard cell culture conditions (ie, a $37^{\circ} \mathrm{C}$, humidified, $5 \% \mathrm{CO}_{2}-95 \%$ air environment). Before experiments, all substrates were immersed in 5 of $\mathrm{mL}$ media in 6-well culture plates. RAEC were lifted from cell culture plates using trypsin/ethylenediaminetetraacetic acid (EDTA) and counted for seeding using a hemacytometer. For cell adhesion experiments, RAEC were seeded at a density of 4500 cells $/ \mathrm{cm}^{2}$ onto the substrates of interest to this study. Cells were allowed to adhere onto the substrates for 5 hours under standard cell culture conditions. Cell proliferation experiments were conducted similarly for 1 and 5 day durations. The media was changed every other day during the proliferation assay. At the end of each cell experiment, all non-adherent cells were removed from the substrate surfaces by washing with PBS. Adherent cells were fixed with a $4 \%$ formaldehyde solution and nuclei stained using Hoechst 33258 dye (Sigma).

Data was collected by counting cells under fluorescence microscopy at five random fields for each substrate. The cell adhesion experiment was completed in duplicate and repeated at two different times. Statistical analysis of the data was performed by using student t-tests to determine differences between means ( $p<0.01$ was considered significant).

\section{Results}

SEM images showed a more rough surface for the fine grain NiTi substrate as compared with the coarse grain NiTi (Figure 2). The fine grain NiTi surface was also visibly more compacted with greater percentages of smaller particles. The largest particle size for the fine grain surface was

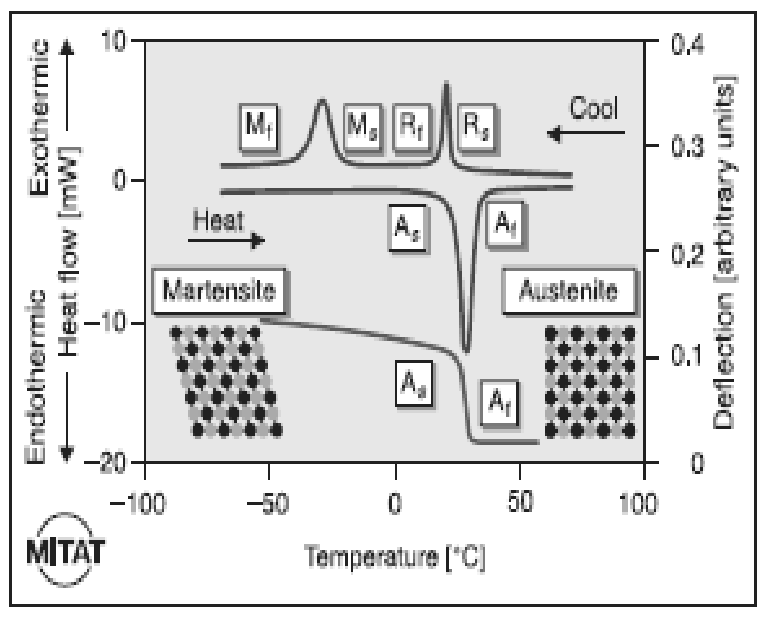

(A)

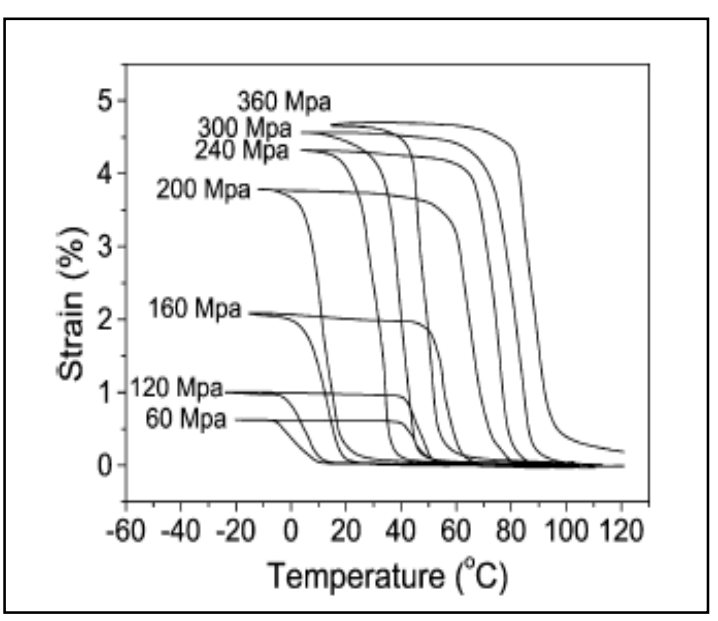

(B)

(Pelton et al 2000)

Figure I A graphical representation of NiTi material properties. The thermal shape memory effect $(\mathbf{A})$ involves the transformation from the austensitic to martensitic phase and vice versa. Permanent deformation is achieved in the martensite phase and below the As transformation temperature. The strain-temperature relation (B) suggests that strain can be achieved as temperature is decreased and this strain is retained if the temperature is kept below As. 


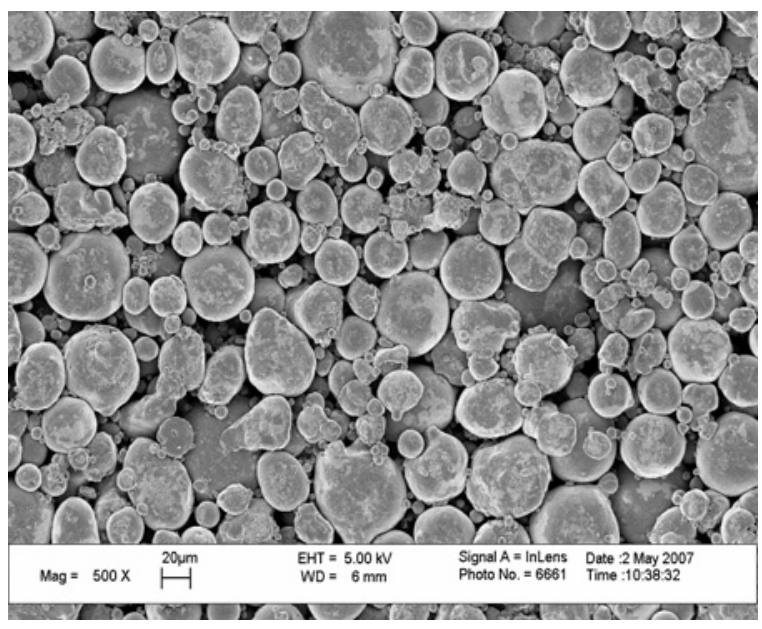

Coarse Grain NiTi (as purchased)

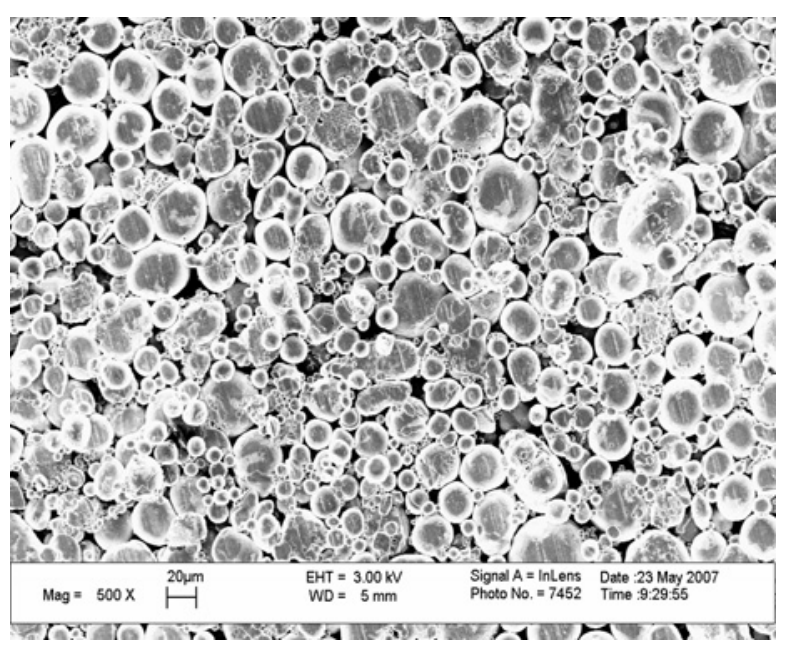

Fine Grain NiTi (with mesh)

Figure 2 SEM images of NiTi compacts with two different surface roughness properties. Compared to the coarse grain NiTi samples, the fine grain NiTi samples had a greater percentage of sub-micron to nanometer size particles and a greater percentage of interparticulate voids providing for increased sub-micron to nanometer roughness.

approximately 50 microns while the smallest particle sizes were less than 1 micron. For the coarse grain surface, the largest particle size was approximately 80 microns and the smallest particles were again less than 1 micron. EDS results confirmed an expected similar surface chemistry between the two substrate types. For example, the fine grain and coarse grain NiTi had an average surface composition of $\mathrm{Ni}=55.1$ wt. $\%$ and 54.5 wt. $\%$, respectively.

Cell experiment results after 5 hours showed that endothelial cells adhered much more $(\mathrm{p}<0.01)$ on the fine grain compared to coarse grain NiTi and convention cp Ti substrates (Figures 3 and 4). Coarse grain NiTi compacts and cp Ti promoted similar levels of endothelial cell adhesion. Interestingly, on the fluorescence microscopy images, it was observed that cells adhered at the NiTi particle boundaries; cell adhesion was greater on the fine grain sample with more surface grain boundaries. The results after 1 day also showed increased endothelial cell density with a decrease in NiTi surface feature dimension into the sub-micron and nanoscale regime (Figures 5 and 6). However, the difference in cell density after 1 day between the fine grain $\mathrm{NiTi}$ and cp Ti was not significant. After 5 days, the cp Ti and the fine grain NiTi substrates were highly confluent whereas the coarse grained NiTi was not.

\section{Discussion}

NiTi has good shape-memory and superelastic properties which makes it an ideal biomaterial for vascular stent applications. The major challenge in vascular stent development is to inhibit or reduce inflammation and thrombogenicity as well as limit conditions that promote neointimal tissue growth. Moreover, stent surfaces are needed that increase the affinity for endothelial cells to form a protective monolayer on the vascular stent surface.

In the present study, we began the investigation of sub-micron to nanostructured surface features on NiTi for improved vascular stent applications. Nanostructured materials (including metals, polymers and ceramics) have already been shown to increase the functions of multiple cell types (including osteoblasts and endothelial cells) (Miller et al 2004; Webster et al 2004; Choudhary et al 2007). Increased cell adhesion on fine grain $(<60 \mu \mathrm{m})$ compared with coarse grain NiTi substrates $(<100 \mu \mathrm{m})$ was observed here. Endothelial cells adhered and proliferated more on NiTi with greater sub-micron and nanoscale surface roughness; such approaches may eliminate the need for anti-thrombogenic and anti-inflammatory drug-eluting stents since this would possibly lead to greater vascular stent endothelialization. In the longer term cell experiments, cells did not grow well on the larger grain size NiTi compacts since the coarse particles created large voids on the surface. However, the presence of a greater amount of homogeneous interparticulate voids on the fine grain $\mathrm{NiTi}$ (and hence, greater porosity at the nanometer scale), seemed more beneficial for endothelial cell adhesion and proliferation leading to the formation of an endothelial cell monolayer as early as 5 days.

Since the present cell experiments were conducted under static conditions, it would also be of value to determine endothelial cell adhesion and migration in flow conditions which simulate the in vivo stent environment. If endothelial 


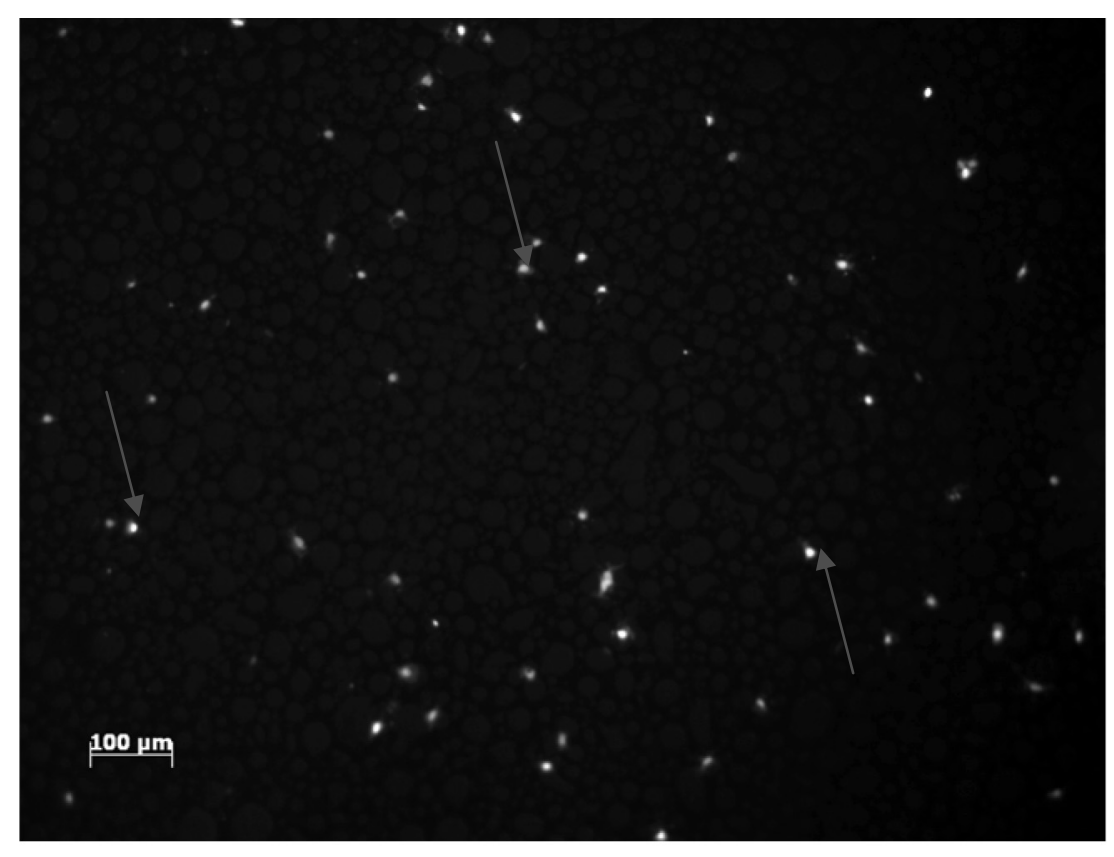

\section{Fine Grain NiTi}
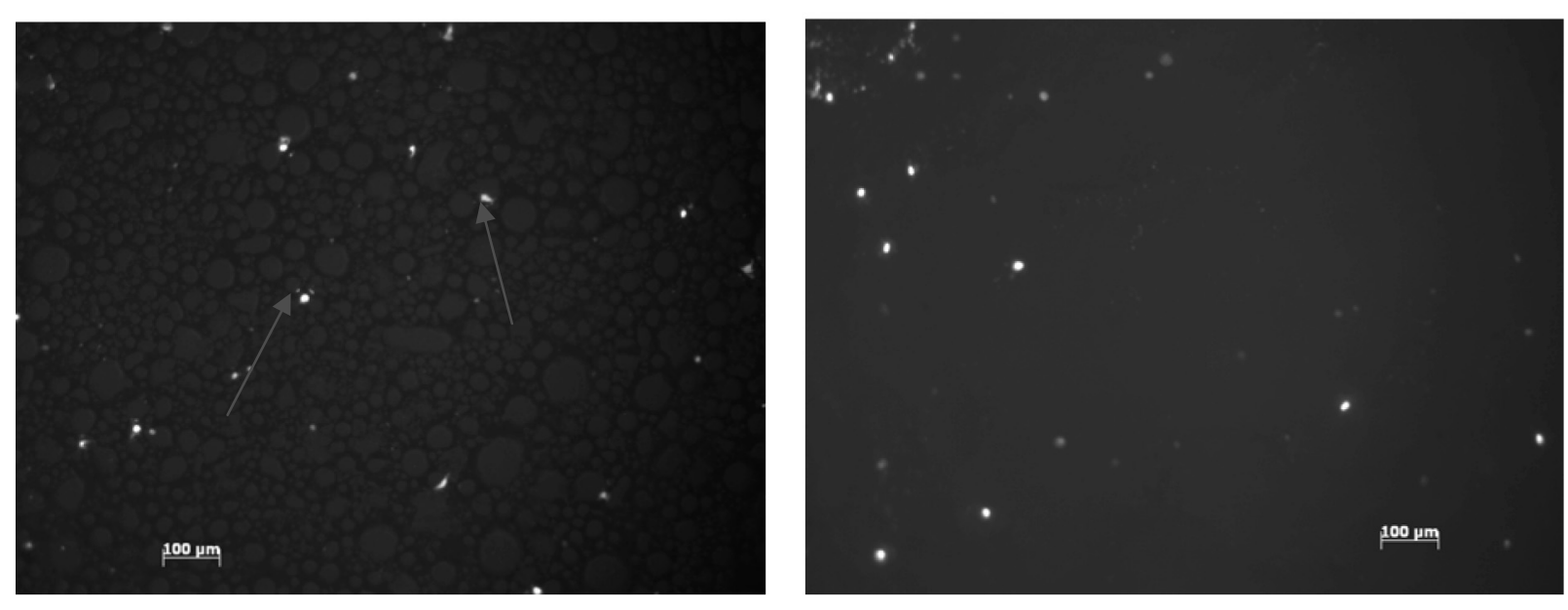

\section{Coarse Grain NiTi}

\section{c.p. Ti}

Figure 3 Fluorescent microscope images of endothelial cell adhesion results showed greater endothelial cell density on the fine grain NiTi substrates as compared to the coarse grain $\mathrm{NiTi}$ and c.p.Ti after 5 hours. Arrows point to endothelial cells.

cells can rapidly adhere and proliferate on sub-micron to nanostructured NiTi, there will be greater protection of the vascular stent surface from any adverse inflammatory and thrombogenic responses. In addition, future studies should focus on whether creating sub-micron to nanometer surface features on other stent materials (for example, stainless steel) would have similar advantages of promoting endothelial cell functions.

In preparing the NiTi substrates, heat treatments were not used for two reasons. First, heat treatments will result in brittle compacts which cannot be used for vascular stent applications. During heat treatments, NiTi is also disposed towards forming a heterogeneous surface oxide layer which makes it vulnerable to corrosion. At room temperature, NiTi is known to oxidize and form a $\mathrm{TiO}_{2}$ film on the surface. If this $\mathrm{TiO}_{2}$ film is uniform across the surface, it may be beneficial for increasing endothelial cell functions (Yeh et al 2006). It is uncertain how our processing of the NiTi substrates in liquid nitrogen may have modified this $\mathrm{TiO}_{2}$ layer. EDS did show that the surface chemistry was very similar for all NiTi substrates; a property 


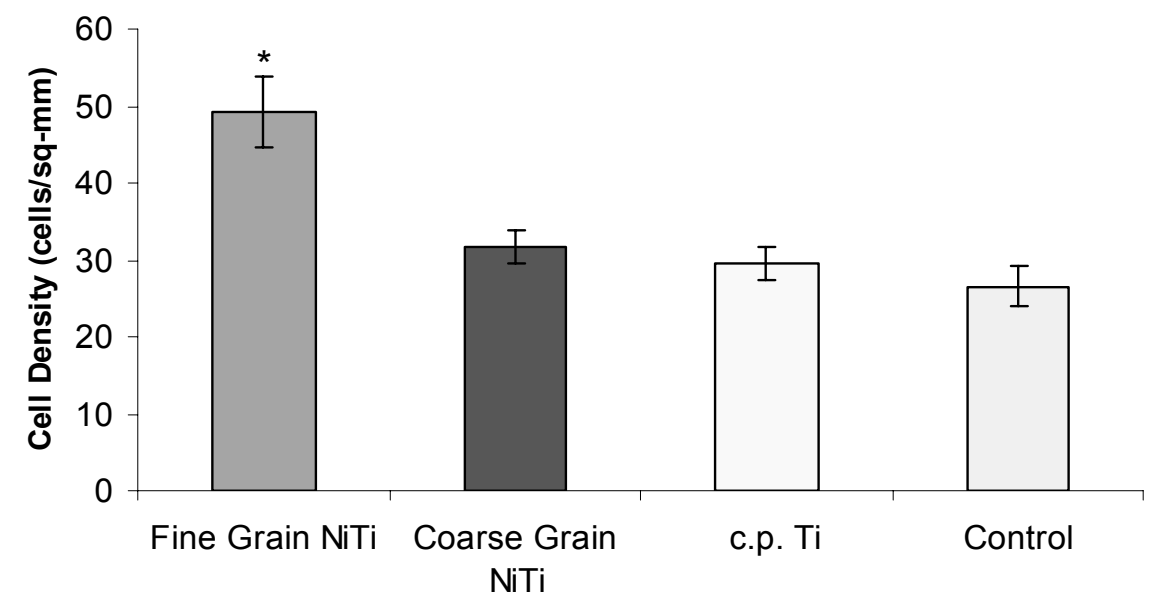

Figure 4 Endothelial cell adhesion increased on fine grain $\mathrm{NiTi}$ substrates with greater submicron to nanometer surface features after 5 hours, ${ }^{*} \mathrm{p}<0.0 \mathrm{I}$ compared to all other substrates. The difference in endothelial cell adhesion between coarse grain NiTi, c.p. Ti, and the control was not significant. $\mathrm{Data}=$ mean $+/-\mathrm{S}$.E.; $\mathrm{N}=3$. Control $=$ tissue culture polystyrene.

important for us to control in order to delineate sub-micron to nanoroughness as a key property towards promoting endothelialization. Importantly, UV light sterilization was used here. It has been reported that exposure of NiTi to steam during autoclaving causes diffusion of $\mathrm{Ni}$ to the surface resulting in higher Ni concentrations (Shabalovskaya et al 2002). Such an event may potentially cause some corrosion in vivo with a release of Ni into the blood stream. It has been implicated in other studies that corrosion is more problematic for nanostructured compared to conventional metals due to the increased presence of grain boundaries at the surface of nanostructured materials (Siegel 1996); this would have to be addressed in future studies for the nanostructured NiTi used here.
NiTi compact synthesis was performed by compressing particles under cold temperatures within a liquid nitrogen bath. Figure 1A suggests that lowering the temperature during compression can achieve a greater percentage of strain or deformation of the NiTi particles. Compressing at higher pressures will increase the austensitic transformation temperature range, and compacts will remain intact at standard cell culture temperatures of $37{ }^{\circ} \mathrm{C}$. Compacts with smaller grain size have higher transformation temperatures as a result of increased local elastic energy due to the presence of more grain boundaries (Xu et al 2004). Although there are potentially numerous ways to create sub-micron to nanostructured NiTi, this study employed simple powder

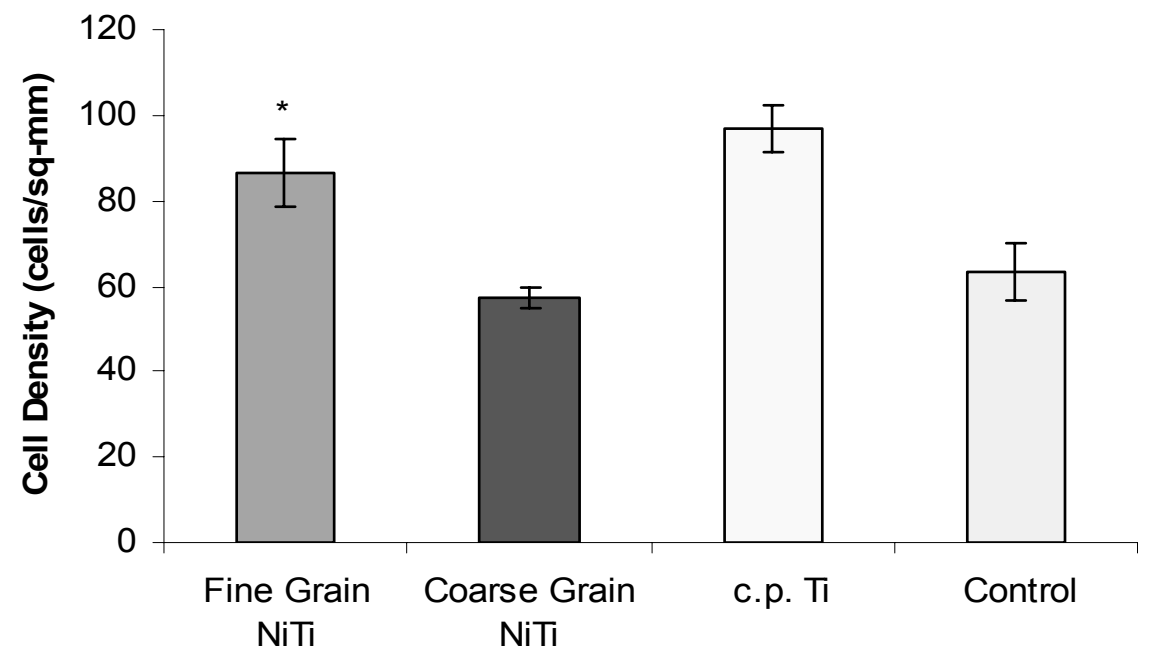

Figure 5 Endothelial cell density increased on fine grain NiTi substrates with greater sub-micron to nanometer surface features after I day. The difference in the number of endothelial cells between fine and coarse grain $\mathrm{NiTi}$ substrates was significant, ${ }^{*} \mathrm{p}<0.0 \mathrm{l}$. However, there was no significant difference in endothelial cell density between fine grain NiTi and c.p. Ti. Data = mean +l- S.E.; $\mathrm{N}=3$. Control $=$ tissue culture polystyrene. 
(A)
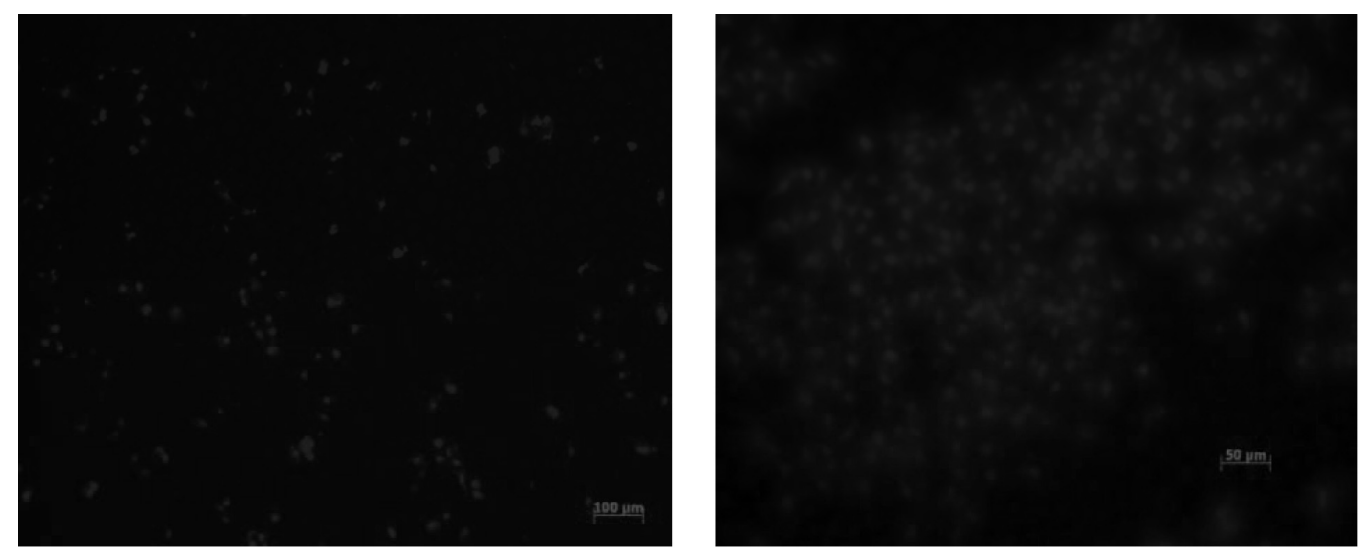

Fine Grain NiTi

(A)

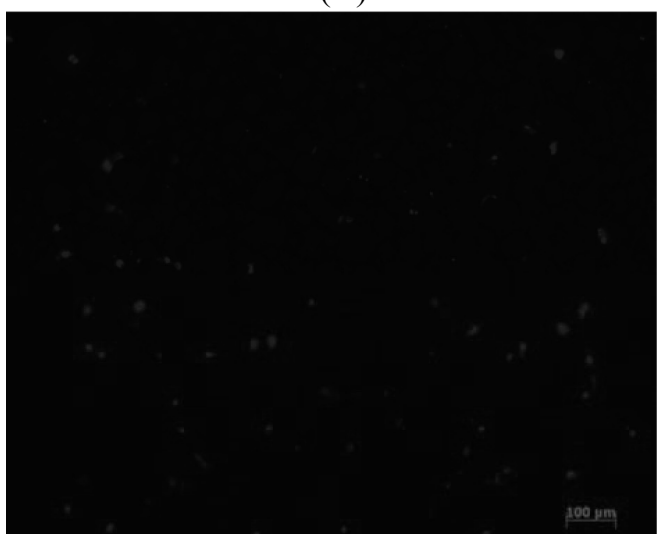

Coarse Grain NiTi

(A)
(B)

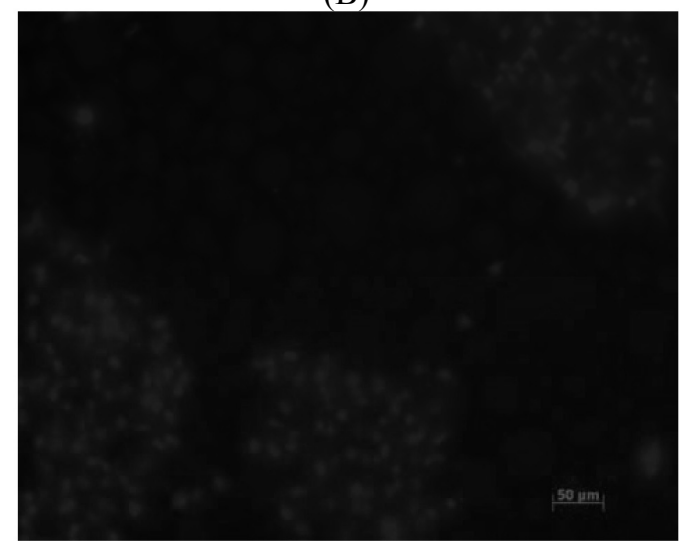

(B)
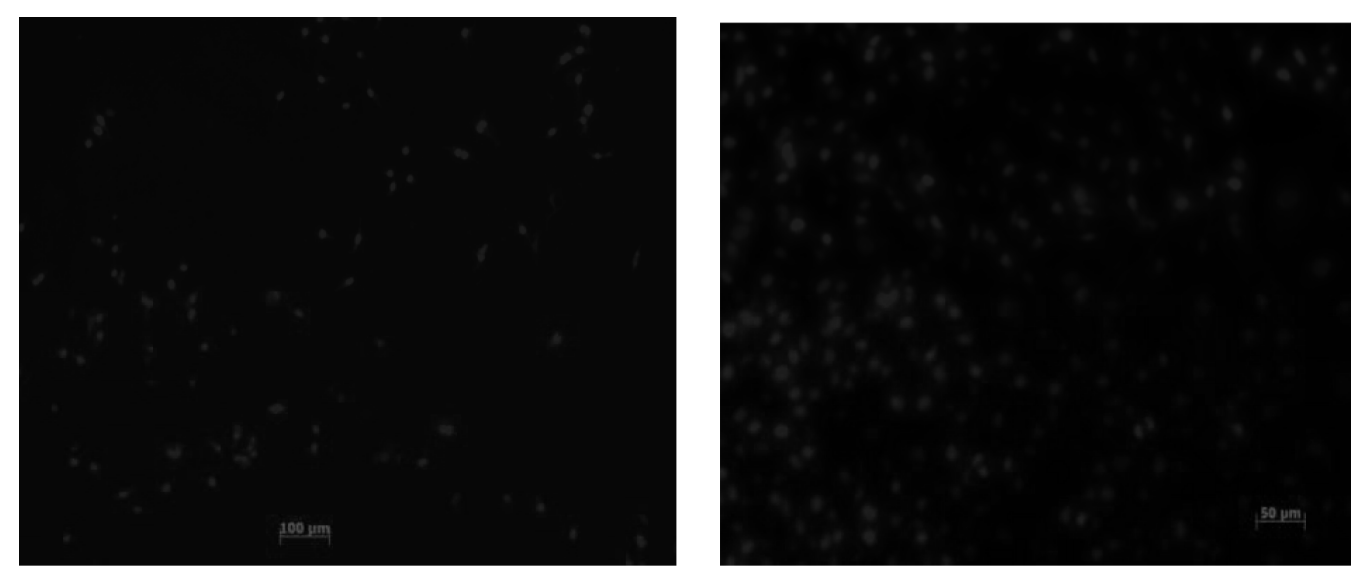

Conventional (c.p.) Ti

Figure 6 Fluorescent microscope images of endothelial cells after $(\mathbf{A})$ I day (Bars $=100 \mu \mathrm{m})$ and $(\mathbf{B}) 5$ days $($ Bars $=50 \mu \mathrm{m})$ on fine and coarse grain NiTi and c.p.Ti substrates. Endothelial cells were more confluent on fine grain NiTi after 5 days compared to coarse grain NiTi. 
metallurgy techniques, and in doing so, it sets the stage for other nanotechnology-based techniques. In summary, the outcome of the present study is consistent with the results of other studies involving nanophase materials (Miller et al 2004; Webster et al 2004; Choudhary et al 2007). This common phenomenon of improved performance using nanostructured materials has drawn much curiosity towards a scientific rationale. An explanation for this phenomenon was given by Klablunde and colleagues (1996), who claimed that nanophase materials have higher numbers of atoms at the surface compared with bulk, greater areas of increased surface defects (such as edge/corner sites and particle boundaries), and larger proportions of surface electron delocalization. These properties have since been shown to modulate initial protein interactions that control subsequent cell functions (Webster et al 2000).

\section{Conclusions}

Here, it was shown that NiTi substrates with greater submicron to nanometer roughness promoted endothelial cell density and monolayer formation. More studies are necessary to corroborate the benefits of nanostructured NiTi for vascular stent applications. Future studies should examine NiTi substrates synthesized with greater percentages of submicron to nanometer roughness to illuminate any additional benefits. Other techniques for synthesizing nanophase NiTi (such as chemically, mechanically or electrically) should also be investigated. Such efforts will facilitate the emergence of a new age of NiTi vascular stents with a greater efficacy at treating atherosclerotic patients than the current failing drugeluting stents. Moreover, these NiTi stents may have superior flexibility and shape-memory benefits which will allow for easier delivery and implantation at the occluded site.

\section{Acknowledgments}

The authors would like to acknowledge Prof Geoffrey Williams (Brown University) for his assistance at the Leduc Imaging Facility, and Mr Charles Vickers (Brown University) for his assistance in the student machine shop.

\section{References}

Armitage DA, Parker TL, Grant DM. 2003. Biocompatibility and hemocompatibility of surface-modified NiTi alloys. J Biomedical Mater Res, 66A:129-37.

Bertrand O, Sipehia R, Mongrain R, et al. 1998. Biocompatibility aspects of new stent technology. J Amer Col Cardiol, 32:562-71.

Choudhary S, Haberstroh KM, Webster TJ. 2007. Enhanced functions of vascular cells on nanostructured Ti for improved stent applications. Tissue Eng, 13:222-7.

Cragg AH, De Jong S, Barnhart WH, et al. 1993. NiTi intravascular stent: results of preclinical evaluation. Radiology, 189:775-8.
Klabunde KJ, Strak J, Koper O, et al. 1996. Nanocrystals as stoichiometric reagents with unique surface chemistry. J Phys Chem, 100:12141.

McDougall J. 2006. The angioplasty debacle. The McDougall Newsletter [online]. 5(9). Accessed on October 15, 2007. URL: http://www. drmcdougall.com/misc/2006nl/sept/angio.htm.

Miller D, Thapa A, Haberstroh KM, et al. 2004. Endothelial and vascular smooth muscle cell function on poly(lactic-co-glycolic acid) with nanostructured surface features. Biomaterials, 25:53-61.

Pelton AR, DiCello J, Miyazaki S. 2000. Optimisation processing and properties of medical grade NiTi wire. Min Invas Ther and Allied Technologies, 9:107-18.

Ryhanen J. 2000. Biocompatibility of NiTi. Min Invas Ther and Allied Technologies, 9:99-106.

Shabalovskaya SA. 2002. Surface, corrosion and biocompatibility aspects of NiTi as an implant material. Biomed Mater and Eng, 12:69-109.

Siegel RW. 1996. Creating nanomaterials. Sci Am, 275:42.

Webster TJ, Ejiofor JU. 2004. Increased osteoblast adhesion on nanophase metals: Ti, Ti6A14V, and CoCrMo. Biomaterials, 25:4731-9.

Webster TJ, Ergun C, Doremus RH, et al. 2000. Specific proteins mediate enhanced osteoblast adhesion on nanophase ceramics. J Biomed Mater Res, 51:475-83.

Wirth C, Comte V, Lagneau C, et al. 2005. NiTi surface roughness modulates in vitro cell response: a comparison between fibroblasts and osteoblasts. Mater Sci Eng C, 25:51-60.

Wu XD, Gun GJ, Wu JS. 2003. The nonlinear relationship between transformation strain and applied stress for NiTi. Mater Lett, 57:1334-8.

$\mathrm{Xu}$ X, Thadhani N. 2004. Shock synthesis and characterization of nanostructured NiTi alloy. Mater Sci Eng A, 384:194-201.

Yeh H, Lu S, Tian T, et al. 2006. Comparison of endothelial cells grown on different stent materials. J Biomed Mater Res, 76A:835-41. 